\title{
REVIEW ARTICLE \\ Systematic review and meta-analysis of the proportion of non-typhoidal Salmonella cases that develop chronic sequelae
}

\author{
J. KEITHLIN ${ }^{1,2}$, J. M. SARGEANT ${ }^{1,2}$, M. K. THOMAS ${ }^{3}$ AND A. FAZIL ${ }^{4}$ \\ ${ }^{1}$ Centre for Public Health and Zoonoses, University of Guelph, Guelph, Ontario, Canada \\ ${ }^{2}$ Department of Population Medicine, Ontario Veterinary College, Guelph, Ontario, Canada \\ ${ }^{3}$ Centre for Food-borne, Environmental and Zoonotic Infectious Diseases, Public Health Agency of Canada, \\ Guelph, Ontario, Canada \\ ${ }^{4}$ Laboratory for Foodborne Zoonoses, Public Health Agency of Canada, Guelph, Ontario, Canada
}

Received 23 April 2014; Final revision 28 August 2014; Accepted 30 September 2014; first published online 30 October 2014

\section{SUMMARY}

The objective of this systematic review and meta-analysis was to estimate the proportion of cases of non-typhoidal salmonellosis (NTS) that develop chronic sequelae, and to investigate factors associated with heterogeneity. Articles published in English prior to July 2011 were identified by searching PubMed, Agricola, CabDirect, and Food Safety and Technology Abstracts. Observational studies reporting the number of NTS cases that developed reactive arthritis (ReA), Reiter's syndrome (RS), haemolytic uraemic syndrome (HUS), irritable bowel syndrome (IBS), inflammatory bowel disease (IBD) or Guillain-Barré syndrome (GBS), Miller-Fisher syndrome (MFS) were included. Metaanalysis was performed using random effects and heterogeneity was assessed using the $I^{2}$ value. Metaregression was used to explore the influence of study-level variables on heterogeneity. A total of 32 studies were identified; 25 reported on ReA, five reported on RS, seven reported on IBS, two reported on IBD, two reported on GBS, one reported on MFS, and two reported on HUS. There was insufficient data in the literature to calculate a pooled estimate for RS, HUS, IBD, GBS, or MFS. The pooled estimate of the proportion of cases of NTS that developed ReA and IBS had substantive heterogeneity, limiting the applicability of a single estimate. Thus, these estimates should be interpreted with caution and reasons for the high heterogeneity should be further explored.

Key words: Inflammatory bowel disease, reactive arthritis, salmonellosis.

\section{INTRODUCTION}

Non-typhoidal Salmonella is an important foodborne pathogen, with an estimated 93.8 million cases and about 155000 deaths globally per year [1]. Common sources of infection include contaminated food, such as meat, eggs and produce [2, 3] and via the

\footnotetext{
* Author for correspondence: Dr J. M. Sargeant, 103 MacNabb House, Ontario Veterinary College, University of Guelph, 50 Stone Rd E., Guelph, Ontario, Canada, N1 G 2 W1. (Email: sargeanj@uoguelph.ca)
}

faecal-oral route after contact with infected animals [4]. Acute symptoms associated with salmonellosis include diarrhoea, fever, headache and abdominal pain. Salmonellosis has also been implicated in the development of chronic sequelae such as reactive arthritis (ReA) [5] and irritable bowel syndrome [6].

Burden of disease (BOD) estimates can be used by researchers and policy makers to help prioritize funding and identify intervention opportunities. Efforts have been made by many countries to estimate the BOD associated with foodborne diseases such as Salmonella (for examples, see [1, 7-9]). There is 
variability in the sequelae included in these estimates and in the sources of data for estimating the frequency of the sequelae [10].

Systematic review and meta-analysis provide an opportunity to assess all of the available literature on a topic in a transparent and reproducible manner, by identifying all of the literature on a topic and combining the results across studies [11]. The purpose of this systematic review and meta-analysis was to estimate the proportion of cases of non-typhoidal salmonellosis (NTS) that develop ReA or Reiter's syndrome (RS), Guillain-Barré syndrome (GBS), Miller Fisher syndrome (MFS), irritable bowel syndrome (IBS), haemolytic uraemic syndrome (HUS), inflammatory bowel disease (IBD) including ulcerative colitis (UC), and Crohn's disease (Crohn's) and to use meta-regression to explore the study-level variables that contributed to variation in estimates. The review was part of a larger project that also estimated the proportion of individuals with E. coli $\mathrm{O} 157$ and Campylobacter who developed chronic sequelae $[12,13]$.

\section{MATERIALS AND METHODS}

\section{Literature search and inclusion-exclusion criteria/data variables}

The literature search was conducted to identify chronic sequelae for multiple foodborne pathogens. The following search terms were entered into four electronic databases (Medline via PubMed, Agricola, CabDirect, and Food Safety and Technology Abstracts); ('Escherichia coli O157', or, 'O157', 'VTEC', 'STEC', 'O157:H7' or Salmonella or Campylobacter) and ('sequel*', 'long-term', 'long term', 'chronic', 'Guillain*', 'HUS', 'hemolytic uremic syndrome', 'haemolytic uraemic syndrome', 'hemorrhagic uremic syndrome', 'haemorrhagic uraemic syndrome', 'Reiter*', 'complication*', 'arthritis', 'irritable bowel syndrome', 'IBS', 'post infectious irritable bowel syndrome' or 'inflammatory bowel disease'), without language restrictions to identify citations from prior to July 2011. The reference lists of relevant studies were reviewed to identify additional studies not located by the initial search. This paper presents results for Salmonella and the chronic sequelae of ReA including RS, IBS, IBD including UC and Crohn's, GBS including MFS, and HUS.

Three levels of screening for eligibility were performed. The first level of screening was performed by a single reviewer using titles and abstracts and excluded references that did not include information on either Campylobacter, E. coli O157 or nontyphoidal Salmonella, did not discuss chronic sequelae in humans or were laboratory-based studies, randomized control trials or reviews. The second level of screening, also based on titles and abstracts, was performed by two independent reviewers with conflicts resolved by consensus. This level restricted results to specific pathogen types (excluding Salmonella typhi and $S$. paratyphi) and the specific chronic sequelae of interest. The final round of screening was performed using the full-text publications and identified studies with the information required to estimate the proportion of NTS cases that developed a chronic sequelae. Those publications that presented the opposite relationship, the number of sequelae cases with evidence of past Salmonella exposure, were excluded.

Full publications that met all eligibility criteria then underwent data extraction. This was performed by two independent reviewers extracting data from each article. Conflicts were resolved via consensus with input from a third reviewer where required. Data were extracted on population (year, country, age range and gender distribution for Salmonella cases), Salmonella species or serotype, study directionality (retrospective $v s$. prospective), source of data (surveillance vs. outbreak vs. hospitalized cases), season and decade of data collection, if sequelae cases were disease negative prior to salmonellosis, categories describing both the Salmonella diagnosis and sequelae diagnosis, the length of time between Salmonella diagnosis and sequelae diagnosis (followup time) and outcomes (number of cases of Salmonella, number of cases of NTS who developed a chronic sequelae). Prospective studies were those where cases of NTS were identified and the assessment for sequelae occurred at a time point in the future. Retrospective studies were those where both the identification as a case of NTS and sequelae diagnosis had already occurred prior to data collection for the study. Diagnosis of Salmonella was categorized as confirmed or probable based on the description of diagnostic methods provided in each publication. Confirmed NTS cases were those where cases were identified by culture, serology or DNA-based tests and probable cases were those identified as a case based on the clinical case definition given in the study. These included self-reported illness from survey data and cases without specific 
diagnostic test results that were linked to outbreaks. Diagnoses of the sequelae were categorized as assessment by specialist, physician diagnosed/taken from medical records, self-reported based on validated scale or self-reported.

The outcome of interest for this systematic review was the proportion of NTS cases that developed a chronic sequela. As some publications reported multiple methods of diagnosing both Salmonella (e.g. a study may have reported both probable and confirmed cases) and the sequelae (e.g. a study may have reported both self-reported and specialist confirmed cases of the sequelae), as well as multiple data sources (e.g. both outbreak-associated and hospitalized cases), it was possible for multiple estimates to be reported from the same study for the proportion of NTS cases that developed a sequela. Therefore the term 'outcome' was used to describe the probability of a case of NTS developing a chronic sequela for each of these various combinations.

\section{Statistical analysis}

For each outcome, the proportion of NTS cases that developed specific chronic sequelae was calculated as the number of persons developing a sequela divided by the total number of NTS cases. Standard errors and confidence intervals for a single proportion were derived. Prior to analysis, to incorporate the influence of study size on the outcome, adjusted proportions and standard errors were calculated using a logit transformation [14].

Meta-analysis was performed for sequelae with more than 10 outcomes using a random-effects model and the DerSimonian \& Laird method to derive the summary estimate [11]. Heterogeneity was assessed using the $I^{2}$ value [15]. To allow for inclusion in the meta-analysis, a count of 0.5 was added or subtracted from the number of sequelae cases in those studies reporting a chronic sequelae outcome of $0 \%$ or $100 \%$, respectively [16]. Meta-regression was used to explore potential sources of heterogeneity if the $I^{2}$ value was higher than $25 \%$ and if more than ten outcomes were present for the sequelae of interest. The source of data (outbreak vs. surveillance vs. hospitalized cases), the method of diagnosing Salmonella, Salmonella serotype, the method used to diagnose the sequelae, disease status prior to illness with Salmonella, country, study directionality, study decade, season, group size and follow-up time were considered as explanatory variables in the meta-regression. All statistical analyses were performed in Stata version 12 (StataCorp, USA).

Categorical variables representing season, decade of study, group size (number of cases of NTS) and follow-up time (time from diagnosis with Salmonella to sequela diagnosis) were generated from the data provided in the studies for use in meta-regression. In the northern hemisphere season was categorized as autumn (September-November), winter (DecemberFebruary), spring (March-May) and summer (June-August). In the southern hemisphere season was classified as autumn (March-May), winter (June-August), spring (September-November) and summer (December-February). Decade of study was classified based on the decade when data collection began. Group size was divided into small $(n \leqslant 100)$, medium $(n=101-1000)$, large $(n=1001-10000)$, and extra-large $(n=>10000)$. Due to the transient nature and varying duration of symptoms associated with many sequelae, follow-up time was divided into three categories; $<3$ months (90 days), $>90$ days to $<1$ year (365 days) and $\geqslant 1$ year (365 days).

Using meta-regression, significance was determined first by univariable analysis $(P \leqslant 0.05$ was considered significant), then significant variables were included in a backwards multivariable model. Meta-regression was performed using logittransformed outcomes and logit-transformed withinstudy standard errors. Those variables that remained significant $(P \leqslant 0 \cdot 05)$ were further explored with subgroup meta-analysis if there were at least two outcomes available in the data for each level of the explanatory variable.

\section{Assessment of factors associated with internal/external validity}

A pre-established risk of bias assessment was not conducted. Alternatively, data were extracted on whether or not information on the following variables was reported in the publication; study directionality (retrospective $v s$. prospective), source of data (outbreak $v s$. surveillance vs. hospitalized cases), method of diagnosis for both Salmonella and for the sequelae, followup time, whether sequelae cases were disease-negative prior to salmonellosis, and population information (country, gender distribution, age range). The definition for sequelae diagnosis was divided into two components; the method of diagnosis (physician diagnosed $v s$. self-reported) and if specific diagnostic criteria were provided. 


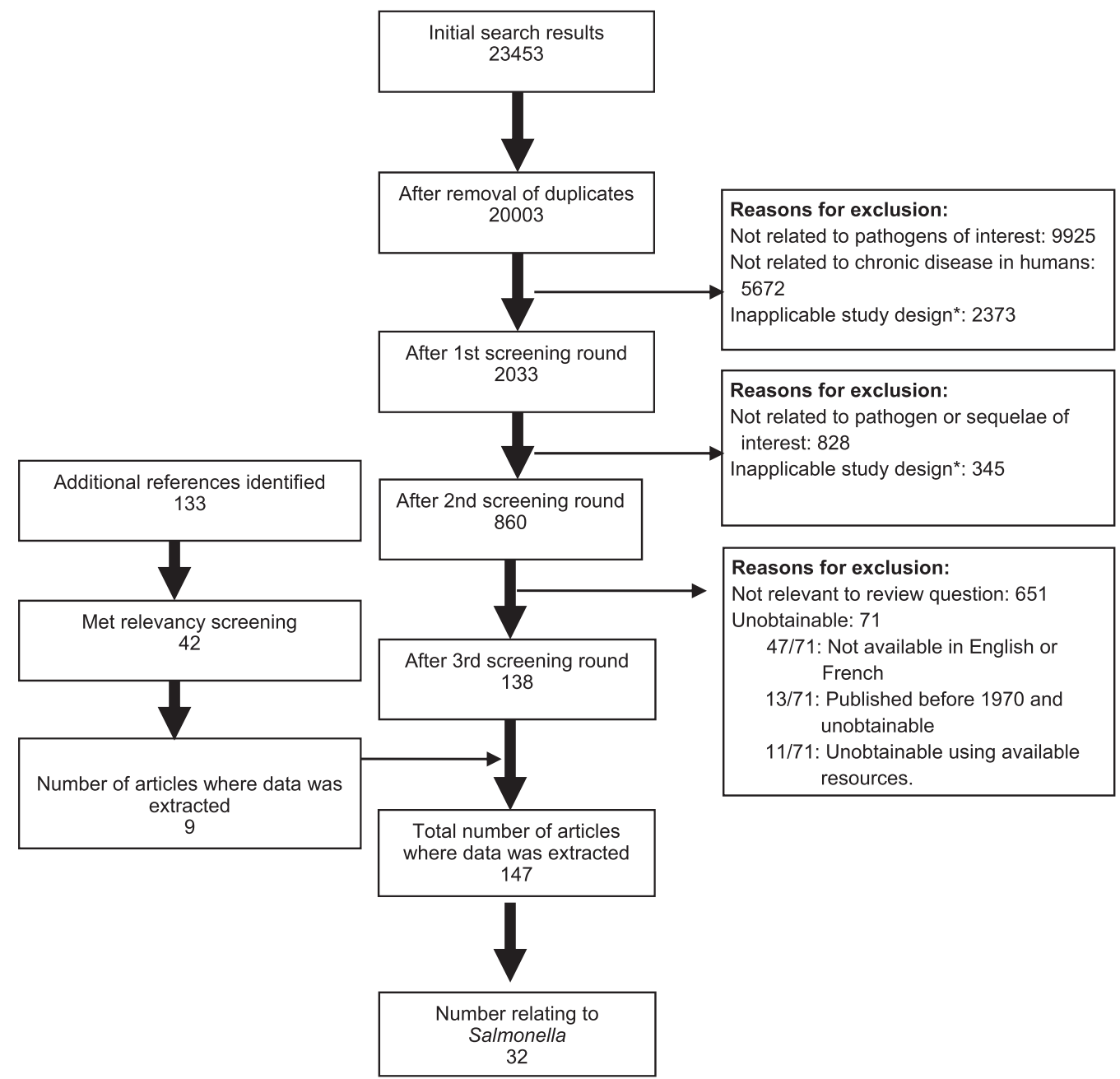

Fig. 1. Flow chart of results from systematic review for Salmonella and chronic sequelae. * Excluded study designs were randomized control trials, laboratory-based studies and those that selected subjects based on sequelae and determined previous Salmonella exposure.

\section{RESULTS}

\section{Systematic review}

\section{Study selection}

The results of the systematic literature search are summarized in Figure 1. After screening and identification of relevant articles from reference lists there were 147 publications that met the inclusion criteria of which 32 contained information on Salmonella and chronic sequelae. Most publications reported on a single sequela, although the number of sequelae reported ranged from 1 to 6 (Table 1).

\section{Reactive arthritis}

\section{Study descriptions}

Of the 32 studies investigating cases of NTS and chronic sequelae (Table 1), 25 provided outcomes for ReA. The 25 studies were from 11 countries, eight of which were European. Seventeen were based on outbreaks and of those that reported source $(n=16)$ all but one were foodborne and 44\% (7/16) of those were attributed to contaminated meat. Of the seven surveillance studies, five were prospective in design. There was only a single study of hospitalized NTS cases. For all studies, follow-up times ranged from 28 to 1080 days (3 years) (Table 2).

\section{Outcomes}

There were 42 outcomes (Table 2), as 13 studies provided multiple outcomes. In all but one of the surveillance-based studies, cases were laboratory confirmed for Salmonella. The number of NTS cases ranged from 61 to 34664 persons, with the probability of a case developing ReA ranging from $0.08 \%$ to 
Table 1. Population characteristics for studies relating to chronic sequelae of Salmonella published before July 2011

\begin{tabular}{|c|c|c|c|c|c|c|c|c|c|}
\hline First author, year [ref.] & Sequelae & Country & $\begin{array}{l}\text { Age range* } \\
\text { (of Salmonella } \\
\text { cases) }\end{array}$ & $\begin{array}{l}\% \text { Female (for } \\
\text { Salmonella } \\
\text { cases) }\end{array}$ & Source of data & $\begin{array}{l}\text { Study } \\
\text { directionality }\end{array}$ & $\begin{array}{l}\text { Outbreak } \\
\text { source }\end{array}$ & $\begin{array}{l}\text { Date for data } \\
\text { collection }\end{array}$ & Season \\
\hline Ternhag, 2008 [21] & $\begin{array}{l}\text { ReA, HUS, IBS, UC, } \\
\text { Crohn's }\end{array}$ & Sweden & All ages & 51 & Surveillance $\dagger$ & Retrospective & n.a. & 1997-2004 & All \\
\hline Doorduyn, 2008 [22] & $\begin{array}{l}\text { ReA, Reiter's, GBS, } \\
\text { MFS, IBS, IBD }\end{array}$ & Netherlands & n.r. & n.r. & Surveillance & Prospective & n.a. & 2002-2003, 2005 & All \\
\hline Ekman, 2000 [23] & $\operatorname{ReA}$ & Finland & Adults & 53 & Surveillance & Prospective & n.a. & 1998-1999 & All \\
\hline Schiellerup, 2008 [24] & $\operatorname{ReA}$ & Denmark & Adults & $57 \cdot 1$ & Surveillance & Prospective & n.a. & $2002-2003$ & All \\
\hline Townes, 2008 [25] & $\operatorname{ReA}$ & USA & All ages & 50 & Surveillance & Prospective & n.a. & 2002-2004 & All \\
\hline Jess, $2011[26]$ & Crohn's, UC & Denmark & All ages & 50 & Surveillance & Retrospective & n.a. & 1992-2008 & All \\
\hline Buxton, 2002 [27] & $\operatorname{ReA}$ & Can.a.da & All ages & 53 & Surveillance & Prospective & n.a. & 1999-2000 & All \\
\hline Arnedo-Pena, 2010 [28] & $\operatorname{ReA}$ & Spain & n.r. & 50 & $\begin{array}{l}\text { Outbreak in } \\
\text { community }\end{array}$ & Prospective & $\begin{array}{r}\text { Food: } \\
\text { meat }\end{array}$ & 2005 & Summer \\
\hline Cowden, 1989 [29] & $\mathrm{UC}$ & England & All ages & 46 & $\begin{array}{l}\text { Outbreak in } \\
\text { community }\end{array}$ & Prospective & $\begin{array}{r}\text { Food: } \\
\text { meat }\end{array}$ & 1987-1988 & Winter \\
\hline Dworkin, 2001 [30] & ReA, Reiter's & USA & Adults & 63 & $\begin{array}{l}\text { Outbreak in } \\
\text { community }\end{array}$ & Prospective & $\begin{array}{r}\text { Food: } \\
\text { meat }\end{array}$ & 1994 & $\begin{array}{c}\text { Autumn/ } \\
\text { winter }\end{array}$ \\
\hline Eastmond, 1983 [31] & $\operatorname{ReA}$ & Scotland & Adults & 50 & $\begin{array}{l}\text { Outbreak in } \\
\text { community }\end{array}$ & Prospective & $\begin{array}{l}\text { Food: } \\
\text { dairy }\end{array}$ & 1981 & Autumn \\
\hline Hakansson, 1975 [32] & $\operatorname{ReA}$ & Sweden & n.r. & n.r. & $\begin{array}{l}\text { Outbreak in } \\
\text { community }\end{array}$ & Prospective & n.r. & 1974 & n.r. \\
\hline Hannu, 2002 [33] & $\operatorname{ReA}$ & Finland & All ages & 59 & $\begin{array}{l}\text { Outbreak in } \\
\text { community }\end{array}$ & Prospective & Other & 1999 & Spring \\
\hline Locht, 2002 [19] & $\operatorname{ReA}$ & Denmark & Adults & 56 & $\begin{array}{l}\text { Outbreak in } \\
\text { community }\end{array}$ & Prospective & $\begin{array}{l}\text { Food: } \\
\text { other }\end{array}$ & 1999 & Winter \\
\hline Locht, 1993 [34] & $\operatorname{ReA}$ & Sweden & Adults & 44 & $\begin{array}{l}\text { Outbreak in } \\
\text { community }\end{array}$ & Prospective & $\begin{array}{l}\text { Food: } \\
\text { other }\end{array}$ & 1990 & Spring \\
\hline Mattila, 1998 [35] & ReA, Reiter's & Finland & All ages & 68 & $\begin{array}{l}\text { Outbreak in } \\
\text { community }\end{array}$ & Prospective & $\begin{array}{l}\text { Food: } \\
\text { vegetable }\end{array}$ & 1994 & Spring \\
\hline Mattila, 1994 [36] & ReA, Reiter's & Finland & All ages & 62 & $\begin{array}{l}\text { Outbreak in } \\
\text { community }\end{array}$ & Prospective & $\begin{array}{l}\text { Food: } \\
\text { vegetable }\end{array}$ & 1992 & Autumn \\
\hline McColl, 2000 [37] & $\operatorname{ReA}$ & Australia & All ages & 51 & $\begin{array}{l}\text { Outbreak in } \\
\text { community }\end{array}$ & Prospective & $\begin{array}{l}\text { Food: } \\
\text { meat }\end{array}$ & 1997 & Autumn \\
\hline McColl, 2000 [37] & $\operatorname{ReA}$ & Australia & All ages & 47 & $\begin{array}{l}\text { Outbreak in } \\
\text { community }\end{array}$ & Prospective & $\begin{array}{l}\text { Food: } \\
\text { meat }\end{array}$ & 1997 & Spring \\
\hline McKendrick, 1994 [38] & IBS & UK & n.r. & 66 & $\begin{array}{l}\text { Outbreak in } \\
\text { community }\end{array}$ & Prospective & $\begin{array}{l}\text { Food: } \\
\text { other }\end{array}$ & Pre-1994 & n.r. \\
\hline Mearin, 2005 [39] & IBS & Spain & Adults & $55 \cdot 3$ & $\begin{array}{l}\text { Outbreak in } \\
\text { community }\end{array}$ & Prospective & $\begin{array}{l}\text { Food: } \\
\text { dairy }\end{array}$ & 2002 & Summer \\
\hline
\end{tabular}


Table 1 (cont.)

\begin{tabular}{|c|c|c|c|c|c|c|c|c|c|}
\hline First author, year [ref.] & Sequelae & Country & $\begin{array}{l}\text { Age range* } \\
\text { (of Salmonella } \\
\text { cases) }\end{array}$ & $\begin{array}{l}\% \text { Female (for } \\
\text { Salmonella } \\
\text { cases) }\end{array}$ & Source of data & $\begin{array}{l}\text { Study } \\
\text { directionality }\end{array}$ & $\begin{array}{l}\text { Outbreak } \\
\text { source }\end{array}$ & $\begin{array}{l}\text { Date for data } \\
\text { collection }\end{array}$ & Season \\
\hline Rudwaleit, 2001 [40] & $\mathrm{ReA}$ & Germany & Youth & n.r. & $\begin{array}{l}\text { Outbreak in } \\
\text { community }\end{array}$ & Prospective & $\begin{array}{l}\text { Food: } \\
\text { dairy }\end{array}$ & 1998 & Winter \\
\hline Samuel, 1995 [41] & $\operatorname{ReA}$ & USA & n.r. & 53 & $\begin{array}{l}\text { Outbreak in } \\
\text { community }\end{array}$ & Prospective & $\begin{array}{l}\text { Food: } \\
\text { meat }\end{array}$ & 1993 & Summer \\
\hline Thomson, 1992 [42] & $\operatorname{ReA}$ & n.r. & Adults & 5 & $\begin{array}{l}\text { Outbreak in } \\
\text { community }\end{array}$ & Prospective & $\begin{array}{l}\text { Food: } \\
\text { meat }\end{array}$ & Pre-1992 & n.r. \\
\hline Urfer, 2000 [43] & ReA, IBS & Switzerland & All ages & 37 & $\begin{array}{l}\text { Outbreak in } \\
\text { community }\end{array}$ & Prospective & $\begin{array}{l}\text { Food: } \\
\text { meat }\end{array}$ & 1993 & Autumn \\
\hline Lee, 2005 [44] & $\operatorname{ReA}$ & Australia & All ages & 48 & $\begin{array}{l}\text { Outbreak in } \\
\text { community }\end{array}$ & Retrospective & $\begin{array}{l}\text { Food: } \\
\text { vegetable }\end{array}$ & 1999 & $\begin{array}{r}\text { Summer/ } \\
\text { autumn }\end{array}$ \\
\hline Thomson, 1995 [20] & ReA/Reiter'st & n.r. & n.r. & n.r. & $\begin{array}{l}\text { Outbreak in } \\
\text { community }\end{array}$ & Retrospective & $\begin{array}{l}\text { Food: } \\
\text { other }\end{array}$ & 1984 & Autumn \\
\hline Rohekar, 2008 [45] & $\operatorname{ReA}$ & Canada & Adults & $71 \cdot 3$ & $\begin{array}{l}\text { Outbreak in } \\
\text { community }\end{array}$ & Retrospective & $\begin{array}{l}\text { Food: } \\
\text { vegetable }\end{array}$ & 2005 & $\begin{array}{l}\text { Autumn/ } \\
\text { winter }\end{array}$ \\
\hline Thomson, 1994 [46] & ReA, Reiter's & n.r. & n.r. & n.r. & $\begin{array}{l}\text { Outbreak in } \\
\text { community }\end{array}$ & Retrospective & $\begin{array}{l}\text { Food: } \\
\text { meat }\end{array}$ & 1990 & Spring \\
\hline Petersen, 1996 [47] & ReA & Denmark & All ages & 49 & $\begin{array}{l}\text { Hospitalized } \\
\text { cases }\end{array}$ & Prospective & n.a. & $1991-1993$ & All \\
\hline Saps, 2008 [48] & IBS & $\begin{array}{l}\text { USA \& } \\
\text { Italy }\end{array}$ & All ages & 48 & $\begin{array}{l}\text { Hospitalized } \\
\text { cases }\end{array}$ & Prospective & n.a. & 2006 & $\begin{array}{r}\text { Summer/ } \\
\text { autumn }\end{array}$ \\
\hline Helms, 2006 [49] & $\begin{array}{l}\text { GBS, IBD, IBS, } \\
\text { HUS, ReA }\end{array}$ & Denmark & All ages & 50 & Surveillance & Retrospective & n.a. & 1991-1999 & All \\
\hline
\end{tabular}

not reported; $\mathrm{n}$ a, not applicable; ReA, reactive arthritis; UC, ulcerative colitis.

$*$ Youth were individuals aged $<18$ years, adults were aged $>18$ years.

$\uparrow$ Surveillance includes laboratory and notifiable disease registries, sporadic cases and other population surveillance.

t Study combined cases of ReA and Reiter's syndrome. 
Table 2. Outcome variables organized by chronic sequelae for studies relating to Salmonella published prior to July 2011

\begin{tabular}{|c|c|c|c|c|c|c|c|c|}
\hline First author, year [ref.] & $\begin{array}{l}\text { Species or } \\
\text { serotype* }\end{array}$ & $\begin{array}{l}\text { Sequelae negative } \\
\text { prior to diagnosis } \\
\text { with Salmonella? }\end{array}$ & $\begin{array}{l}\text { Time from Salmonella } \\
\text { diagnosis to } \\
\text { evaluation for chronic } \\
\text { sequelae (days) }\end{array}$ & $\begin{array}{l}\text { Diagnosis of } \\
\text { Salmonella } \dagger\end{array}$ & $\begin{array}{l}\text { Diagnosis of } \\
\text { sequelae }\end{array}$ & $\begin{array}{l}\text { Number of } \\
\text { people with } \\
\text { Salmonella }\end{array}$ & $\begin{array}{l}\text { Number of } \\
\text { people who } \\
\text { developed } \\
\text { sequelae }\end{array}$ & Outcome \\
\hline \multicolumn{9}{|l|}{$\operatorname{Re} A$} \\
\hline Arnedo-Pena, 2010 [28] & Hadar & $\begin{array}{l}\text { All were disease } \\
\text { negative }\end{array}$ & 90 & Probable & $\begin{array}{l}\text { Disease status } \\
\text { confirmed by } \\
\text { specialist }\end{array}$ & 155 & 13 & $8 \cdot 4 \%$ \\
\hline Arnedo-Pena, 2010 [28] & Hadar & $\begin{array}{l}\text { All were disease } \\
\text { negative }\end{array}$ & 120 & Probable & $\begin{array}{l}\text { Self-reported based } \\
\text { on validated scale }\end{array}$ & 155 & 16 & $10 \cdot 3 \%$ \\
\hline Arnedo-Pena, 2010 [28] & Hadar & $\begin{array}{l}\text { All were disease } \\
\text { negative }\end{array}$ & 150 & Confirmed & $\begin{array}{l}\text { Disease status } \\
\text { confirmed by } \\
\text { specialist }\end{array}$ & 67 & 6 & $9 \cdot 0 \%$ \\
\hline Buxton, 2002 [27] & Typhimurium & $\begin{array}{l}\text { All were disease } \\
\text { negative }\end{array}$ & 90 & Confirmed & $\begin{array}{l}\text { Disease status } \\
\text { confirmed by } \\
\text { specialist }\end{array}$ & 61 & 4 & $6 \cdot 6 \%$ \\
\hline Buxton, 2002 [27] & Typhimurium & $\begin{array}{l}\text { All were disease } \\
\text { negative }\end{array}$ & 120 & Confirmed & $\begin{array}{l}\text { Self-reported based } \\
\text { on validated scale }\end{array}$ & 66 & 17 & $25 \cdot 8 \%$ \\
\hline Doorduyn, 2008 [22] & n.r. & n.r. & 1080 & Confirmed & Self-reported & 181 & 8 & $4 \cdot 4 \%$ \\
\hline Dworkin, 2001 [30] & Enteritidis & n.r. & 30 & Probable & Self-reported & 217 & 63 & $29 \cdot 0 \%$ \\
\hline Eastmond, 1983 [31] & Typhimurium & n.r. & 60 & Confirmed & $\begin{array}{l}\text { Medical records/ } \\
\text { physiciant }\end{array}$ & 418 & 8 & $1 \cdot 9 \%$ \\
\hline Ekman, 2000 [23] & n.r. & $\begin{array}{l}\text { All were disease } \\
\text { negative }\end{array}$ & n.r. & Confirmed & $\begin{array}{l}\text { Disease status } \\
\text { confirmed by } \\
\text { specialist }\end{array}$ & 198 & 8 & $4 \cdot 0 \%$ \\
\hline Ekman, 2000 [23] & n.r. & $\begin{array}{l}\text { All were disease } \\
\text { negative }\end{array}$ & n.r. & Confirmed & Self-reported & 198 & 13 & $6 \cdot 6 \%$ \\
\hline Hakansson, 1975 [32] & Typhimurium & n.r. & n.r. & Probable & n.r. & 330 & 13 & $3 \cdot 9 \%$ \\
\hline Hannu, 2002 [33] & Typhimurium & $\begin{array}{l}\text { All were disease } \\
\text { negative }\end{array}$ & 60 & Confirmed & $\begin{array}{l}\text { Disease status } \\
\text { confirmed by } \\
\text { specialist }\end{array}$ & 63 & 5 & $7 \cdot 9 \%$ \\
\hline Helms, 2006 [49] & Combined & $\begin{array}{l}\text { All were disease } \\
\text { negative }\end{array}$ & 365 & Confirmed & $\begin{array}{l}\text { Medical records/ } \\
\text { physician }\end{array}$ & 27894 & 87 & $0 \cdot 3 \%$ \\
\hline Helms, 2006 [49] & Enteritidis & $\begin{array}{l}\text { All were disease } \\
\text { negative }\end{array}$ & 365 & Confirmed & $\begin{array}{l}\text { Medical records/ } \\
\text { physician }\end{array}$ & 14533 & 50 & $0 \cdot 3 \%$ \\
\hline Helms, 2006 [49] & Typhimurium & $\begin{array}{l}\text { All were disease } \\
\text { negative }\end{array}$ & 365 & Confirmed & $\begin{array}{l}\text { Medical records/ } \\
\text { physician }\end{array}$ & 7021 & 24 & $0 \cdot 3 \%$ \\
\hline Helms, 2006 [49] & Other & $\begin{array}{l}\text { All were disease } \\
\text { negative }\end{array}$ & 365 & Confirmed & $\begin{array}{l}\text { Medical records/ } \\
\text { physician }\end{array}$ & 6340 & 13 & $0 \cdot 2 \%$ \\
\hline
\end{tabular}


Table 2 (cont.)

\begin{tabular}{|c|c|c|c|c|c|c|c|c|}
\hline First author, year [ref.] & $\begin{array}{l}\text { Species or } \\
\text { serotype* }\end{array}$ & $\begin{array}{l}\text { Sequelae negative } \\
\text { prior to diagnosis } \\
\text { with Salmonella? }\end{array}$ & $\begin{array}{l}\text { Time from Salmonella } \\
\text { diagnosis to } \\
\text { evaluation for chronic } \\
\text { sequelae (days) }\end{array}$ & $\begin{array}{l}\text { Diagnosis of } \\
\text { Salmonella }\end{array}$ & $\begin{array}{l}\text { Diagnosis of } \\
\text { sequelae }\end{array}$ & $\begin{array}{l}\text { Number of } \\
\text { people with } \\
\text { Salmonella }\end{array}$ & $\begin{array}{l}\text { Number of } \\
\text { people who } \\
\text { developed } \\
\text { sequelae }\end{array}$ & Outcome \\
\hline Lee, 2005 [44] & Typhimurium & $\begin{array}{l}\text { All were disease } \\
\text { negative }\end{array}$ & 90 & Confirmed & $\begin{array}{l}\text { Medical records/ } \\
\text { physician }\end{array}$ & 261 & 38 & $14 \cdot 6 \%$ \\
\hline Lee, 2005 [44] & Typhimurium & $\begin{array}{l}\text { All were disease } \\
\text { negative }\end{array}$ & 120 & Confirmed & $\begin{array}{l}\text { Medical records/ } \\
\text { physician }\end{array}$ & 54 & 13 & $24 \cdot 0 \%$ \\
\hline Lee, 2005 [44] & Typhimurium & $\begin{array}{l}\text { All were disease } \\
\text { negative }\end{array}$ & 150 & Confirmed & $\begin{array}{l}\text { Medical records/ } \\
\text { physician }\end{array}$ & 207 & 25 & $12 \cdot 1 \%$ \\
\hline Locht, 2002 [19] & n.r. & No - excluded§ & 28 & Probable & Self-reported & 91 & 17 & $18 \cdot 7 \%$ \\
\hline Locht, 1993 [34] & Enteritidis & $\begin{array}{l}\text { All were disease } \\
\text { negative }\end{array}$ & 30 & Probable & Self-reported & 108 & 17 & $15 \cdot 7 \%$ \\
\hline Locht, 1993 [34] & Enteritidis & $\begin{array}{l}\text { All were disease } \\
\text { negative }\end{array}$ & 30 & Confirmed & Self-reported & 89 & 16 & $18 \cdot 0 \%$ \\
\hline Mattila, 1998 [35] & Bovismorbificans & $\begin{array}{l}\text { All were disease } \\
\text { negative }\end{array}$ & 90 & Confirmed & $\begin{array}{l}\text { Disease status } \\
\text { confirmed by } \\
\text { specialist }\end{array}$ & 191 & 22 & $11 \cdot 5 \%$ \\
\hline Mattila, 1994 [36] & n.r. & $\begin{array}{l}\text { All were disease } \\
\text { negative }\end{array}$ & 150 & Confirmed & $\begin{array}{l}\text { Disease status } \\
\text { confirmed by } \\
\text { specialist }\end{array}$ & 246 & 16 & $6 \cdot 5 \%$ \\
\hline McColl, 2000 [37] & Typhimurium & $\begin{array}{l}\text { All were disease } \\
\text { negative }\end{array}$ & 90 & Probable & Self-reported & 312 & 13 & $4 \cdot 2 \%$ \\
\hline McColl, 2000 [37] & Typhimurium & $\begin{array}{l}\text { All were disease } \\
\text { negative }\end{array}$ & 90 & Probable & Self-reported & 112 & 6 & $5 \cdot 4 \%$ \\
\hline Petersen, 1996 [47] & Enteritidis & n.r. & n.r. & Confirmed & $\begin{array}{l}\text { Medical records/ } \\
\text { Physician }\end{array}$ & 48 & 4 & $8 \cdot 3 \%$ \\
\hline Petersen, 1996 [47] & Typhimurium & n.r. & n.r. & Confirmed & $\begin{array}{l}\text { Medical records/ } \\
\text { Physician }\end{array}$ & 40 & 3 & $7 \cdot 5 \%$ \\
\hline Petersen, 1996 [47] & Other & n.r. & n.r. & Confirmed & $\begin{array}{l}\text { Medical records/ } \\
\text { Physician }\end{array}$ & 128 & 7 & $5 \cdot 5 \%$ \\
\hline Rohekar, 2008 [45] & Enteritidis & n.r. & n.r. & Confirmed & Self-reported & 104 & 65 & $62 \cdot 5 \%$ \\
\hline Rudwaleit, 2001 [40] & Enteritidis & $\begin{array}{l}\text { All were disease } \\
\text { negative }\end{array}$ & 120 & Probable & Combination & 286 & $0 \cdot 5$ & $0 \cdot 2 \%$ \\
\hline Samuel, 1995 [41] & n.r. & n.r. & 196 & Probable & $\begin{array}{l}\text { Medical records/ } \\
\text { physician }\end{array}$ & 321 & 23 & $7 \cdot 2 \%$ \\
\hline Schiellerup, 2008 [24] & Other & No-excluded & 30 & Confirmed & $\begin{array}{l}\text { Self-reported based } \\
\text { on validated scale }\end{array}$ & 619 & 104 & $16 \cdot 8 \%$ \\
\hline Schiellerup, 2008 [24] & Typhimurium & No - excluded & 30 & Confirmed & $\begin{array}{l}\text { Self-reported based } \\
\text { on validated scale }\end{array}$ & 193 & 29 & $15 \cdot 0 \%$ \\
\hline
\end{tabular}


Table 2 (cont.)

\begin{tabular}{|c|c|c|c|c|c|c|c|c|}
\hline First author, year [ref.] & $\begin{array}{l}\text { Species or } \\
\text { serotype* }\end{array}$ & $\begin{array}{l}\text { Sequelae negative } \\
\text { prior to diagnosis } \\
\text { with Salmonella? }\end{array}$ & $\begin{array}{l}\text { Time from Salmonella } \\
\text { diagnosis to } \\
\text { evaluation for chronic } \\
\text { sequelae (days) }\end{array}$ & $\begin{array}{l}\text { Diagnosis of } \\
\text { Salmonella }\end{array}$ & $\begin{array}{l}\text { Diagnosis of } \\
\text { sequelae }\end{array}$ & $\begin{array}{l}\text { Number of } \\
\text { people with } \\
\text { Salmonella }\end{array}$ & $\begin{array}{l}\text { Number of } \\
\text { people who } \\
\text { developed } \\
\text { sequelae }\end{array}$ & Outcome \\
\hline Schiellerup, 2008 [24] & Enteritidis & No - excluded & 30 & Confirmed & $\begin{array}{c}\text { Self-reported based } \\
\text { on validated scale }\end{array}$ & 270 & 49 & $18 \cdot 2 \%$ \\
\hline Schiellerup, 2008 [24] & Other & No- excluded & 30 & Confirmed & $\begin{array}{c}\text { Self-reported based } \\
\text { on validated scale }\end{array}$ & 156 & 26 & $16 \cdot 7 \%$ \\
\hline Ternhag, 2008 [21] & n.r. & n.r. & 365 & n.r. & $\begin{array}{l}\text { Medical records/ } \\
\text { physician }\end{array}$ & 34664 & 27 & $0 \cdot 08 \%$ \\
\hline Thomson, 1992 [42] & Heidelberg & $\begin{array}{l}\text { All were disease } \\
\text { negative }\end{array}$ & 30 & Probable & Self-reported & 73 & 6 & $8 \cdot 2 \%$ \\
\hline Thomson, 1994 [46] & Enteritidis & $\begin{array}{l}\text { All were disease } \\
\text { negative }\end{array}$ & 60 & Probable & $\begin{array}{l}\text { Medical records/ } \\
\text { physician }\end{array}$ & 29 & 8 & $27 \cdot 6 \%$ \\
\hline Townes, 2008 [25] & n.r. & $\begin{array}{l}\text { All were disease } \\
\text { negative }\end{array}$ & 42 & Confirmed & Combination & 1356 & 17 & $1 \cdot 3 \%$ \\
\hline $\begin{array}{l}\text { Urfer, } 2000 \text { [43] } \\
\text { Reiter's }\end{array}$ & Braenderup & n.r. & 180 & Probable & Self-reported & 156 & 1 & $0 \cdot 6 \%$ \\
\hline Dworkin, 2001 [30] & Enteritidis & n.r. & 30 & Probable & $\begin{array}{l}\text { Meet study } \\
\text { definition based on } \\
\text { self-reported } \\
\text { symptoms }\end{array}$ & 217 & 6 & $2 \cdot 7 \%$ \\
\hline Doorduyn, 2008 [22] & n.r. & n.r. & 1080 & DNA based & Self-reported & 193 & 0 & $0 \%$ \\
\hline Mattila, 1998 [35] & Bovismorbificans & $\begin{array}{l}\text { All were disease } \\
\text { negative }\end{array}$ & 90 & Culture & $\begin{array}{l}\text { Disease status } \\
\text { confirmed by } \\
\text { specialist }\end{array}$ & 191 & 0 & $0 \%$ \\
\hline Mattila, 1994 [36] & Enterica & $\begin{array}{l}\text { All were disease } \\
\text { negative }\end{array}$ & 150 & Culture & $\begin{array}{l}\text { Disease status } \\
\text { confirmed by } \\
\text { specialist }\end{array}$ & 246 & 0 & $0 \%$ \\
\hline Thomson, 1994 [46] & Enteritidis & $\begin{array}{l}\text { All were disease } \\
\text { negative }\end{array}$ & 60 & Probable & Physician diagnosed & 29 & 2 & $6 \cdot 9 \%$ \\
\hline $\begin{array}{l}\text { ReA/Reiter's combined } \\
\text { Thomson, } 1995 \text { [20] }\end{array}$ & Typhimurium & $\begin{array}{l}\text { All were disease } \\
\text { negative }\end{array}$ & 90 & Probable & $\begin{array}{c}\text { Self-reported based } \\
\text { on validated scale }\end{array}$ & 423 & 27 & $6 \cdot 4 \%$ \\
\hline GBS & & & & & & & & \\
\hline Helms, 2006 [49] & Enteritidis & $\begin{array}{l}\text { All were disease } \\
\text { negative }\end{array}$ & 365 & Confirmed & $\begin{array}{l}\text { Medical records/ } \\
\text { physician }\end{array}$ & 14533 & 1 & $0 \cdot 01 \%$ \\
\hline Helms, 2006 [49] & Typhimurium & $\begin{array}{l}\text { All were disease } \\
\text { negative }\end{array}$ & 365 & Confirmed & $\begin{array}{l}\text { Medical records/ } \\
\text { physician }\end{array}$ & 7021 & 1 & $0 \cdot 01 \%$ \\
\hline
\end{tabular}


Table 2 (cont.)

\begin{tabular}{|c|c|c|c|c|c|c|c|c|}
\hline First author, year [ref.] & $\begin{array}{l}\text { Species or } \\
\text { serotype* }\end{array}$ & $\begin{array}{l}\text { Sequelae negative } \\
\text { prior to diagnosis } \\
\text { with Salmonella? }\end{array}$ & $\begin{array}{l}\text { Time from Salmonella } \\
\text { diagnosis to } \\
\text { evaluation for chronic } \\
\text { sequelae (days) }\end{array}$ & $\begin{array}{l}\text { Diagnosis of } \\
\text { Salmonella } \dagger\end{array}$ & $\begin{array}{l}\text { Diagnosis of } \\
\text { sequelae }\end{array}$ & $\begin{array}{l}\text { Number of } \\
\text { people with } \\
\text { Salmonella }\end{array}$ & $\begin{array}{l}\text { Number of } \\
\text { people who } \\
\text { developed } \\
\text { sequelae }\end{array}$ & Outcome \\
\hline Helms, 2006 [49] & Other & $\begin{array}{l}\text { All were disease } \\
\text { negative }\end{array}$ & 365 & Confirmed & $\begin{array}{l}\text { Medical records/ } \\
\text { physician }\end{array}$ & 6340 & 2 & $0 \cdot 03 \%$ \\
\hline $\begin{array}{l}\text { Doorduyn, } 2008 \text { [22] } \\
\text { MFS }\end{array}$ & n.r. & n.r. & 1080 & Confirmed & Self-reported & 193 & 0 & $0 \cdot 00 \%$ \\
\hline $\begin{array}{l}\text { Doorduyn, } 2008 \text { [22] } \\
\text { IBS }\end{array}$ & n.r. & n.r. & 1080 & Confirmed & Self-reported & 193 & 0 & $0 \cdot 00 \%$ \\
\hline Ternhag, 2008 [21] & Spp. & n.r. & 365 & . & $\begin{array}{l}\text { Medical records/ } \\
\text { physician }\end{array}$ & 34664 & 5 & $0 \cdot 01 \%$ \\
\hline Helms, 2006 [49] & Combined & $\begin{array}{l}\text { All were disease } \\
\text { negative }\end{array}$ & 365 & Confirmed & $\begin{array}{l}\text { Medical records/ } \\
\text { physician }\end{array}$ & 27894 & 252 & $0 \cdot 9 \%$ \\
\hline Helms, 2006 [49] & Enteritidis & $\begin{array}{l}\text { All were disease } \\
\text { negative }\end{array}$ & 365 & Confirmed & $\begin{array}{l}\text { Medical records/ } \\
\text { physician }\end{array}$ & 14533 & 125 & $0 \cdot 9 \%$ \\
\hline Helms, 2006 [49] & Typhimurium & $\begin{array}{l}\text { All were disease } \\
\text { negative }\end{array}$ & 365 & Confirmed & $\begin{array}{l}\text { Medical records/ } \\
\text { physician }\end{array}$ & 7021 & 67 & $1 \cdot 0 \%$ \\
\hline Helms, 2006 [49] & Other & $\begin{array}{l}\text { All were disease } \\
\text { negative }\end{array}$ & 365 & Confirmed & $\begin{array}{l}\text { Medical records/ } \\
\text { physician }\end{array}$ & 6340 & 60 & $1 \cdot 0 \%$ \\
\hline Doorduyn, 2008 [22] & n.r. & No - included§ & 1080 & Confirmed & Self-reported & 193 & 12 & $6 \cdot 2 \%$ \\
\hline Saps, 2008 [48] & n.r. & n.r. & 180 & Confirmed & Self-reported & 24 & 9 & $37 \cdot 5 \%$ \\
\hline Urfer, 2000 [43] & Braenderup & n.r. & 180 & Probable & Self-reported & 156 & 12 & $7 \cdot 7 \%$ \\
\hline McKendrick, 1994 [38] & Enteritidis & $\begin{array}{l}\text { All were disease } \\
\text { negative }\end{array}$ & 365 & Probable & $\begin{array}{l}\text { Self-reported based } \\
\text { on validated scale }\end{array}$ & 38 & 12 & $31 \cdot 6 \%$ \\
\hline Mearin, 2005 [39] & Enteritidis & No - excluded & 90 & Probable & $\begin{array}{l}\text { Self-reported based } \\
\text { on validated scale }\end{array}$ & 367 & 27 & $7 \cdot 4 \%$ \\
\hline Mearin, 2005 [39] & Enteritidis & No - excluded & 180 & Probable & $\begin{array}{l}\text { Self-reported based } \\
\text { on validated scale }\end{array}$ & 341 & 37 & $10 \cdot 9 \%$ \\
\hline Mearin, 2005 [39] & Enteritidis & No - excluded & 365 & Probable & $\begin{array}{l}\text { Self-reported based } \\
\text { on validated scale }\end{array}$ & 266 & 31 & $11 \cdot 7 \%$ \\
\hline \multicolumn{9}{|l|}{ IBD } \\
\hline Helms, 2006 [49] & Combined & $\begin{array}{l}\text { All were disease } \\
\text { negative }\end{array}$ & 365 & Confirmed & $\begin{array}{l}\text { Medical records/ } \\
\text { physician }\end{array}$ & 27894 & 125 & $0 \cdot 5 \%$ \\
\hline Helms, 2006 [49] & Enteritidis & $\begin{array}{l}\text { All were disease } \\
\text { negative }\end{array}$ & 365 & Confirmed & $\begin{array}{l}\text { Medical records/ } \\
\text { physician }\end{array}$ & 14533 & 50 & $0 \cdot 3 \%$ \\
\hline Helms, 2006 [49] & Typhimurium & $\begin{array}{l}\text { All were disease } \\
\text { negative }\end{array}$ & 365 & Confirmed & $\begin{array}{l}\text { Medical records/ } \\
\text { physician }\end{array}$ & 7021 & 47 & $0 \cdot 7 \%$ \\
\hline Helms, 2006 [49] & Other & $\begin{array}{l}\text { All were disease } \\
\text { negative }\end{array}$ & 365 & Confirmed & $\begin{array}{l}\text { Medical records/ } \\
\text { physician }\end{array}$ & 6340 & 28 & $0 \cdot 4 \%$ \\
\hline
\end{tabular}


Table 2 (cont.)

\begin{tabular}{|c|c|c|c|c|c|c|c|c|}
\hline First author, year [ref.] & $\begin{array}{l}\text { Species or } \\
\text { serotype* }\end{array}$ & $\begin{array}{l}\text { Sequelae negative } \\
\text { prior to diagnosis } \\
\text { with Salmonella? }\end{array}$ & $\begin{array}{l}\text { Time from Salmonella } \\
\text { diagnosis to } \\
\text { evaluation for chronic } \\
\text { sequelae (days) }\end{array}$ & $\begin{array}{l}\text { Diagnosis of } \\
\text { Salmonella }\end{array}$ & $\begin{array}{l}\text { Diagnosis of } \\
\text { sequelae }\end{array}$ & $\begin{array}{l}\text { Number of } \\
\text { people with } \\
\text { Salmonella }\end{array}$ & $\begin{array}{l}\text { Number of } \\
\text { people who } \\
\text { developed } \\
\text { sequelae }\end{array}$ & Outcome \\
\hline Doorduyn, 2008 [22] & n.r. & No - included & 1080 & Confirmed & $\begin{array}{l}\text { Self-reported disease } \\
\text { status }\end{array}$ & 193 & 12 & $6 \cdot 2 \%$ \\
\hline \multicolumn{9}{|l|}{ Crohn's } \\
\hline Ternhag, 2008 [21] & spp. & n.r. & 365 & n.r. & $\begin{array}{l}\text { Medical records/ } \\
\text { physician }\end{array}$ & 34664 & 14 & $0 \cdot 04 \%$ \\
\hline Jess, 2011 [26] & Combined & $\begin{array}{l}\text { All were disease } \\
\text { negative }\end{array}$ & up to 16 years & Confirmed & $\begin{array}{l}\text { Medical records/ } \\
\text { physician }\end{array}$ & 41628 & 78 & $0 \cdot 2 \%$ \\
\hline \multicolumn{9}{|l|}{$\mathbf{U C}$} \\
\hline Ternhag, 2008 [21] & spp. & n.r. & 365 & n.r. & $\begin{array}{l}\text { Medical records/ } \\
\text { physician }\end{array}$ & 34664 & 29 & $0 \cdot 08 \%$ \\
\hline Jess, 2011 [26] & Combined & $\begin{array}{l}\text { All were disease } \\
\text { negative }\end{array}$ & up to 16 years & Confirmed & $\begin{array}{l}\text { Medical records/ } \\
\text { physician }\end{array}$ & 41628 & 264 & $0 \cdot 6 \%$ \\
\hline Cowden,1989 [29] & Typhimurium & n.r. & n.r. & Confirmed & n.r. & 85 & 1 & $1 \cdot 2 \%$ \\
\hline
\end{tabular}

GBS, Guillain-Barré syndrome; IBD, inflammatory bowel disease; IBS, irritable bowel syndrome; MFS, Miller Fisher syndrome; n.r., not reported; ReA, reactive arthritis;

UC, ulcerative colitis.

* Combined = study combined non-typhoid strains; Other = non-typhoid strains other than Enteritidis and Typhimurium.

$\dagger$ Confirmed for Salmonella included those confirmed by culture, DNA-based tests and serology. Probable includes those identified as a case of non-typhoidal salmonellosis based on clinical case definition from study.

$\$$ Medical records/physician included those hospitalized for sequelae or diagnosed by a physician.

$\S$ No - excluded: cases of non-typhoidal salmonellosis with previous medical history of related sequelae were excluded from analysis. No - included: cases of non-typhoidal salmonellosis with previous medical history of related sequelae were not excluded from analysis. 


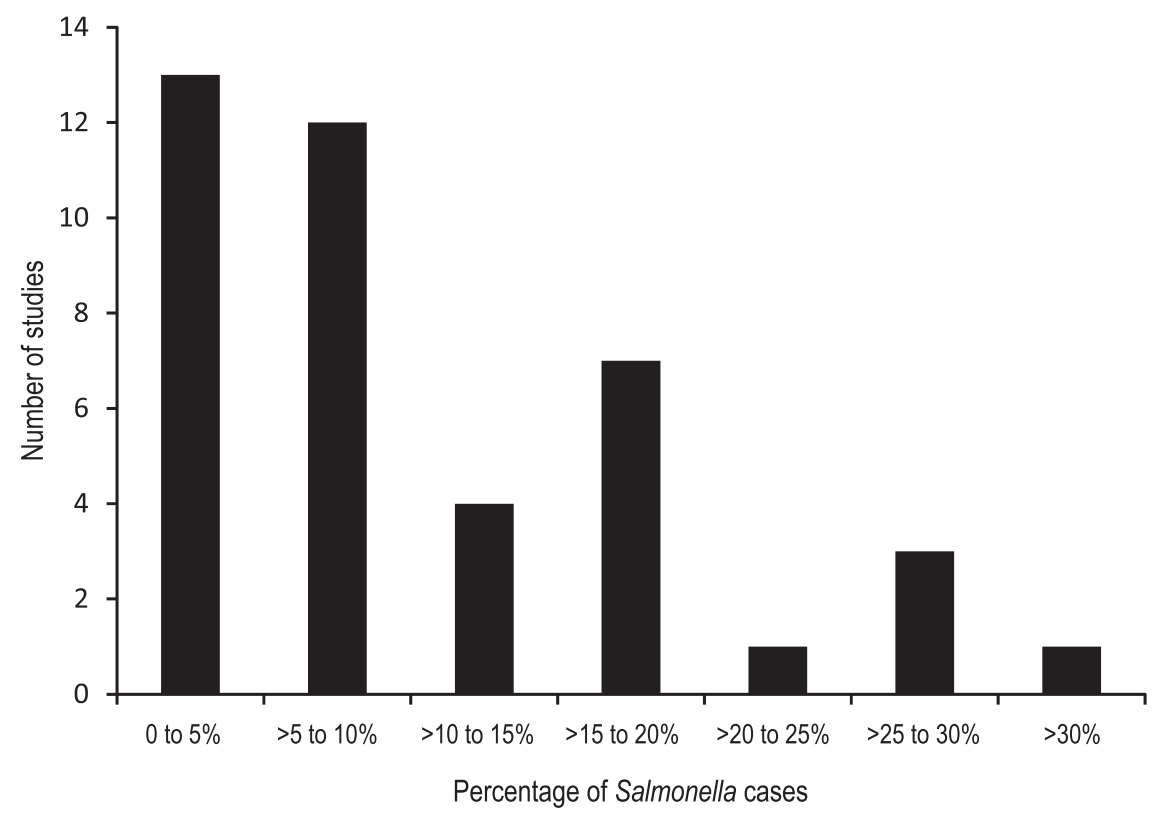

Fig. 2. Distribution of proportions of non-typhoidal salmonellosis cases that developed reactive arthritis from 32 studies published prior to July 2011.

$25 \cdot 8 \%$. For outbreak studies, confirmed NTS cases ranged from 63 to 418 cases with the proportion of cases developing ReA varying from $1 \cdot 9 \%$ to $62 \cdot 5 \%$. The case numbers for probable NTS cases from outbreaks ranged from 29 to 330 persons with $0 \cdot 2$ $29.0 \%$ of cases developing ReA. Overall, most studies reported estimates of $<10 \%$ of NTS cases developing ReA (Fig. 2).

\section{Assessment of factors associated with internal or external validity}

The majority $(84 \%, 21 / 25)$ of studies reported the time from Salmonella diagnosis to diagnosis for ReA. Nine studies $(36 \%)$ did not report whether or not individuals were negative for ReA prior to illness with Salmonella. Age range and gender distribution of NTS cases were not reported in 24\% (6/25) and 16\% $(4 / 25)$ of studies, respectively. Data source (surveillance $v s$. hospitalized cases vs. outbreak), study directionality (retrospective vs. prospective), and method for sequelae diagnosis were reported in all studies. The method for how Salmonella was diagnosed was not reported in a single study. The specific diagnostic criteria used in sequelae diagnosis were not reported in six studies.

\section{Meta-analysis/meta-regression}

A total of 42 outcomes were included in the meta-analysis for Salmonella and ReA. The overall estimate of the proportion of NTS cases that developed ReA was $5 \cdot 8 \%$ [ $95 \%$ confidence interval (CI) $3 \cdot 2-10 \cdot 3, I^{2}=98 \cdot 7 \%$ (Fig. 3). Because of the high heterogeneity, meta-regression was used to explore potential sources.

Variables significantly contributing to heterogeneity in univariable analysis were group size $(P<0 \cdot 0001)$, country $(P=0.046)$, source of data $(P=0.014)$, follow-up time $(P<0.001)$ and sequelae diagnosis $(P=0.013)$ (Table 3$)$. In the multivariable analysis, only group size remained significant $(P=0.037)$ (Table 4). However, group size is not a factor that could directly impact the outcome, and there was considerable correlation between group size and the other, more biologically plausible, variables considered in the univariable analysis (data not shown). Therefore, the multivariable model was re-run excluding the group size variable. Both follow-up time $(P=0 \cdot 001)$ and method of sequelae diagnosis $(P=0.045)$ remained significant.

Subgroup meta-analysis estimated the proportion of NTS cases that developed ReA as $12 \cdot 0 \%$ (95\% CI $\left.7 \cdot 7-18 \cdot 2, I^{2}=94 \%\right)$ in studies where follow-up occurred within 90 days compared to $0.4 \%$ (95\% CI $0 \cdot 2-0 \cdot 7, I^{2}=95 \cdot 40 \%$ ) in those that occurred $\geqslant 1$ year after Salmonella infection (Table 4). In a separate subgroup meta-analysis, the method of diagnosis was significant with rates of ReA estimated as 7.7\% (95\% CI $5 \cdot 9-10 \cdot 1, I^{2}=27 \cdot 1 \%$ ) in cases confirmed by a specialist, $2 \cdot 6 \%\left(95 \%\right.$ CI $\left.0 \cdot 9-7 \cdot 3, I^{2}=98 \cdot 9 \%\right)$ in cases 


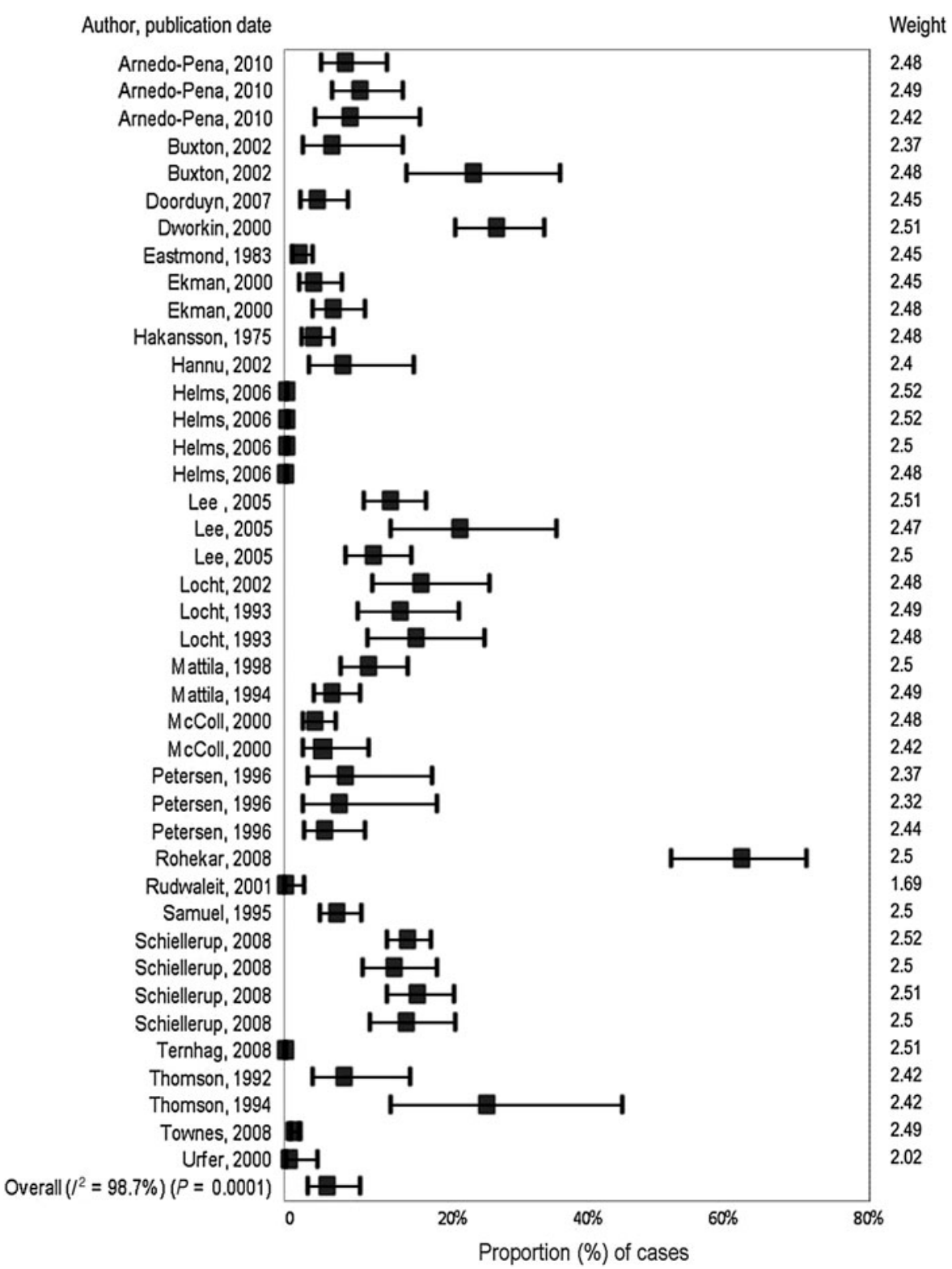

Fig. 3. Forest plot from meta-analysis of cases of non-typhoidal salmonellosis and reactive arthritis for studies published prior to July 2011.

identified by medical records, $16 \cdot 6 \%(95 \%$ CI $14 \cdot 0$ $\left.19 \cdot 7, I^{2}=44 \cdot 5 \%\right)$ in those that were self-reported using a validated scale and $11 \cdot 1 \%(95 \%$ CI $5 \cdot 5-21 \cdot 1$, $\left.I^{2}=95 \cdot 3 \%\right)$ in self-reported cases.

\section{Reiter's syndrome}

RS is related to ReA and is diagnosed based on a triad of symptoms; arthritis in combination with conjunctivitis and urethritis [6]. Five studies, each with one outcome, were examined for Salmonella and RS. Four studies were based on outbreaks and the proportion of RS ranged from $0 \%$ to $6 \cdot 90 \%$. One publication presented ReA and RS in combination, with the proportion of cases that developed either sequelae equal to $6 \cdot 6 \%$. Due to insufficient data, a summary proportion was not calculated.

\section{ReA and RS combined}

The study by Thomson et al. [20] included cases of RS in their definition for ReA. Due to the inconsistent case definition, these results were not included in the meta-analysis for ReA (Table 2). 
Table 3. Summary of variables explored in meta-analysis by sequelae

\begin{tabular}{lll}
\hline \hline Sequelae & $\begin{array}{l}\text { Study/population variables explored with } \\
\text { univariable analysis }\end{array}$ & $\begin{array}{l}\text { Study/population variables explored with } \\
\text { multivariable analysis }\end{array}$ \\
\hline Reactive arthritis & $\begin{array}{l}\text { Time from Salmonella diagnosis to evaluation for } \\
\text { chronic sequelae (follow-up time) }\end{array}$ & $\begin{array}{l}\text { Group size } \\
\text { Country }\end{array}$ \\
& Salmonella species or serotype & Source of data \\
& $\begin{array}{l}\text { Diagnosis of Salmonella (probable } v s . \\
\text { confirmed) }\end{array}$ & $\begin{array}{l}\text { Follow-up time } \\
\text { Diagnosis of sequelae }\end{array}$ \\
& Method of sequelae diagnosis & \\
& Source of data & \\
& Study directionality & \\
& Season & \\
& Country & Group size \\
Irritable bowel & Group size & Country \\
syndrome & Time from Salmonella diagnosis to evaluation for \\
& chronic sequelae (follow-up time) & Source of data \\
& Salmonella species or serotype & Study directionality \\
& Diagnosis of Salmonella (probable vs. & Method of sequelae diagnosis \\
& confirmed) & \\
Method of sequelae diagnosis & \\
& Source of data & \\
Study directionality & \\
& Season & \\
Country & Group size & \\
\hline \hline
\end{tabular}

Table 4. Subgroup meta-analyses by group size, follow-up time and sequelae diagnosis for Salmonella and reactive arthritis

\begin{tabular}{|c|c|c|c|c|}
\hline Variable & $\begin{array}{l}\text { Summary } \\
\text { estimate }\end{array}$ & $(95 \% \mathrm{CI})$ & $I^{2}$ & $\begin{array}{l}\text { Number of } \\
\text { observations }\end{array}$ \\
\hline Overall estimate & $5 \cdot 8 \%$ & $(3 \cdot 2-10 \cdot 3)$ & $98 \cdot 7 \%$ & 42 \\
\hline \multicolumn{5}{|l|}{ Group size } \\
\hline Small & $14 \cdot 2 \%$ & $(10 \cdot 3-19 \cdot 4)$ & $62 \cdot 7 \%$ & 11 \\
\hline Medium & $9 \cdot 0 \%$ & $(6 \cdot 4-12 \cdot 7)$ & $93 \cdot 6 \%$ & 24 \\
\hline Large & $0 \cdot 5 \%$ & $(0 \cdot 2-1 \cdot 2)$ & $92 \cdot 8 \%$ & 3 \\
\hline Extra large & $0 \cdot 2 \%$ & $(0 \cdot 09-0 \cdot 5)$ & $95 \cdot 7 \%$ & 3 \\
\hline \multicolumn{5}{|l|}{ Follow-up time, days } \\
\hline$<90$ & $12 \cdot 0 \%$ & $(7 \cdot 7-18 \cdot 2)$ & $94 \%$ & 13 \\
\hline 90 days to $<365$ & $9 \cdot 0 \%$ & $(6 \cdot 5-12 \cdot 3)$ & $80 \cdot 6 \%$ & 15 \\
\hline$\geqslant 365$ & $0 \cdot 4 \%$ & $(0 \cdot 2-0 \cdot 7)$ & $95 \cdot 4 \%$ & 6 \\
\hline \multicolumn{5}{|l|}{ Sequelae diagnosis } \\
\hline Specialist & $7 \cdot 7 \%$ & $(5 \cdot 9-10 \cdot 1)$ & $27 \cdot 1 \%$ & 7 \\
\hline Physician diagnosed/medical records & $2 \cdot 6 \%$ & $(0 \cdot 9-7 \cdot 3)$ & $98 \cdot 9 \%$ & 14 \\
\hline Self-reported based on validated scale & $16 \cdot 6 \%$ & $(14 \cdot 0-19 \cdot 7)$ & $44 \cdot 5 \%$ & 6 \\
\hline Self-reported & $11 \cdot 1 \%$ & $(5 \cdot 5-21 \cdot 1)$ & $95 \cdot 3$ & 11 \\
\hline
\end{tabular}

CI, Confidence interval.

\section{Irritable bowel syndrome}

\section{Study descriptions}

Seven studies provided information on Salmonella and IBS (Table 1). The studies were from seven different European countries, with one of the studies presenting both US and Italian data. Design types included outbreak (3/7) and surveillance (3/7) studies with one study of hospitalized cases. The majority $(71 \%, 5 / 7)$ were prospective and follow-up times ranged from 3 months (90 days) to 3 years (1080 days) (Table 2 ). 


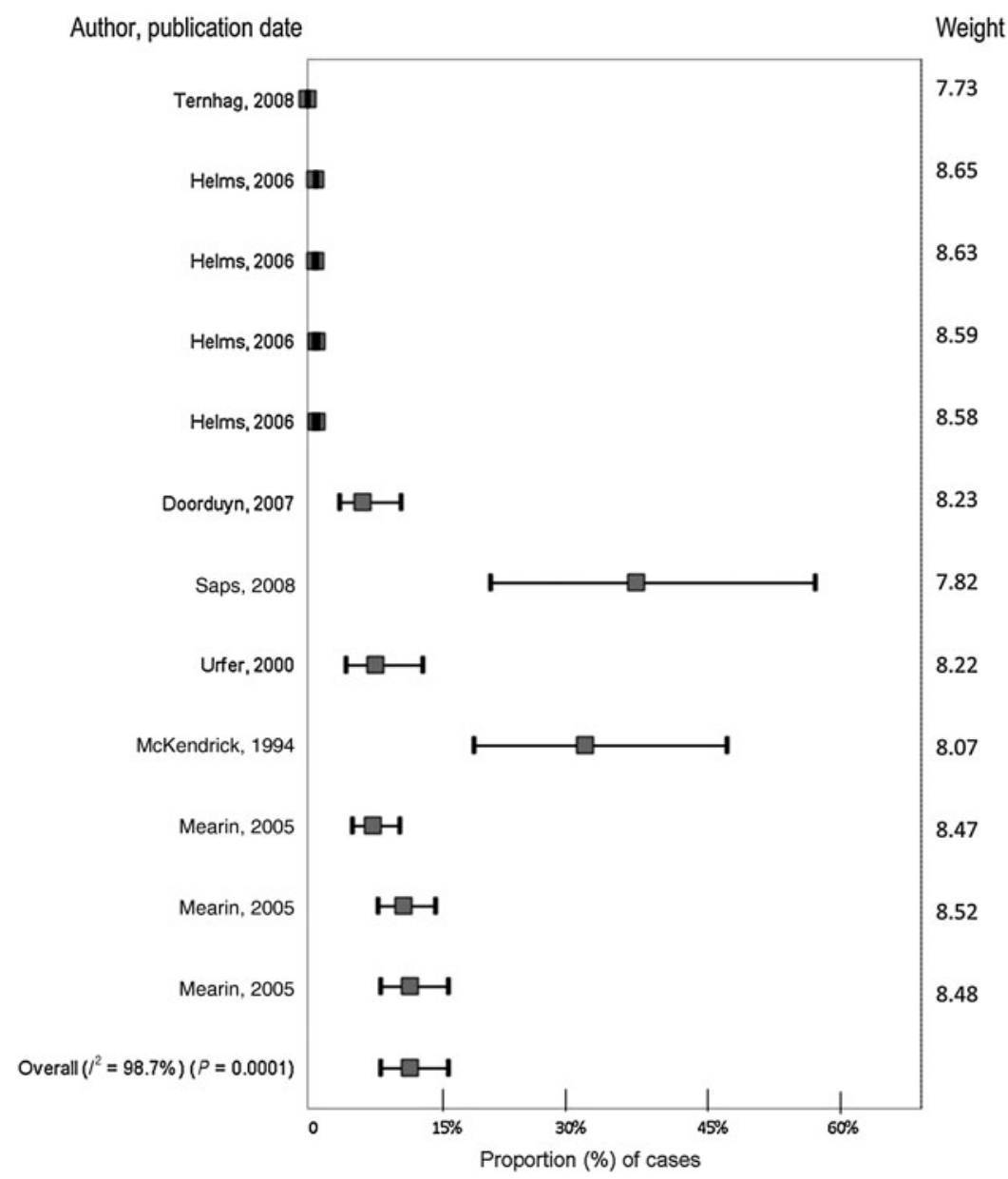

Fig. 4. Forest plot from meta-analysis of cases of non-typhoidal salmonellosis and IBS from studies published prior to July 2011.

\section{Outcomes}

There were 12 outcomes, as two studies [39, 49] reported multiple outcomes (Table 2). For surveillance-based studies, the number of NTS cases varied from 193 and 34664 . All were confirmed NTS cases and the proportion of NTS cases developing IBS ranged from $0 \cdot 01 \%$ to $6 \cdot 2 \%$. Outbreak studies ranged from 38 to 367 cases of NTS of which none were confirmed for Salmonella and IBS occurred in $7 \cdot 4 \%$ to $31 \cdot 6 \%$ of cases.

\section{Assessment of factors associated with internal or external validity}

Data on age range of the NTS cases was not reported in any study and two studies did not report information on gender distribution of NTS cases. Study directionality and source of data were reported in all studies. The method of diagnosis for Salmonella was not reported in any publication. Follow-up time was reported in all studies but three studies did not report on whether all Salmonella cases were disease negative for IBS prior to the onset of Salmonella. All studies reported the method of diagnosing IBS (physician vs. self-reported); however, the specific diagnostic criteria used were not reported in four of the studies.

\section{Meta-analysis/meta-regression}

Twelve outcomes were included in the analysis. The estimate for the proportion of NTS cases that developed IBS was $3 \cdot 3 \%\left(95 \%\right.$ CI $\left.1 \cdot 6-6 \cdot 6, \quad I^{2}=98 \cdot 7\right)$ (Fig. 4). In univariable analyses, group size $(P<$ $0 \cdot 001)$, country $(P=0.006)$, source of data $(P=$ $0 \cdot 014)$, study directionality $(P=0 \cdot 001)$, method of IBS diagnosis $(P=0.008)$ significantly contributed to the heterogeneity in the data (Table 3). Age range of NTS cases $(P=0.057)$ was close to the pre-specified significant cut-point. Due to the potential biological significance and implications in BOD measures it 
was included in the model. In a multivariable analysis, all variables remained significant except for group size. Due to the limited number of studies, subgroup meta-analyses of these factors was not possible.

\section{IBD including Crohn's disease and UC}

IBD includes Crohn's disease and UC, which share similar symptomology and pathogenesis but are considered separate diseases [17]. Two studies reported a total of five different outcomes for Salmonella and IBD (Table 2). All were surveillance studies of confirmed cases of NTS with the proportion of NTS cases developing IBD ranging from $0 \cdot 3 \%$ to $6 \cdot 2 \%$. Two studies reported results for Crohn's disease. Both were large retrospective studies where the occurrence of Crohn's disease was $0 \cdot 04 \%$ and $0 \cdot 2 \%$. Three studies reported on the proportion of NTS cases that developed UC. Estimates ranged from $0 \cdot 08 \%$ in cultureconfirmed cases of NTS to $1.2 \%$ in those not reporting how Salmonella was diagnosed. Due to insufficient data, a summary proportion was not calculated.

\section{Guillain-Barré syndrome}

Two studies provided four outcomes for Salmonella and GBS. Both were surveillance studies with GBS occurring in $0 \%$ and $0.03 \%$ of confirmed NTS cases (Table 2). Due to insufficient data, a summary proportion was not calculated.

\section{Miller Fisher syndrome}

MFS is an uncommon variant of GBS, which is characterized by ophthalmoplegia, ataxia and areflexia [18]. One prospective surveillance study from The Netherlands reported results for MFS. None of the 193 NTS cases developed MFS (Table 2). As there was only a single outcome, meta-analysis was not performed.

\section{Haemolytic uraemic syndrome}

Two studies reported a total of five different outcomes for Salmonella and HUS (Table 2). Both were large retrospective surveillance studies with HUS reported in $0.003 \%$ and $0.016 \%$ of cases. Due to insufficient data, a summary proportion was not calculated.

\section{DISCUSSION}

In this study, the results of a systematic literature search and meta-analysis were used to estimate the proportion of NTS cases that developed ReA, and IBS. A range of sequelae was considered in this review; although not all of these sequelae are commonly associated with Salmonella they were included as they have been associated with other foodborne pathogens. There were insufficient data to calculate summary proportions for the other sequelae considered in this review. A comprehensive literature search of multiple databases was conducted making it probable that this review captured the majority of research available on the proportion of NTS cases that develop these chronic sequelae. Contributing to the limited number of studies on chronic sequelae could be the issue of under-reporting and under-diagnosis of Salmonella [8], as links between infection and sequelae development would not be evaluated on unidentified cases. In addition, when specific sequelae were not reported, it was not possible to determine whether they were not evaluated or whether they were evaluated but not identified in any of the cases.

Although data were limited for some of the sequelae of interest, estimates were calculated for ReA and IBS. Similar to results reported for Campylobacter [12], group size and follow-up time were significantly associated with the proportion of NTS cases that developed ReA. The lower proportion of ReA reported in larger study sizes could be due to differences between study types in case definition, case ascertainment, or intensity of follow-up. Stratifying estimates within study type (e.g. large population studies and outbreak studies) would potentially result in more consistent estimates; however, there were a small number of studies for each design type.

Although all our results indicate that the proportion of cases developing ReA was higher when evaluated within 90 days, evidence of arthritis lasting over 5 years post-infection exists [20]. The higher proportions observed with shorter follow-up time could be attributed to whether studies were capturing incident or prevalent cases and three scenarios could potentially explain this finding; the publications captured a high number of incident cases of short disease duration (within 90 days), others could be capturing remaining prevalent cases that were incident cases within first 3 months and remained symptomatic, while others could be capturing incident cases that developed after 90 days or a combination of these. From a BOD perspective, clarification surrounding disease progression and duration is important considering the relative impact on health systems of a high number of cases of ReA of short duration vs. a low number of 
chronic cases. As these two situations may require different resources and incur different costs, there is the potential need to separate them when calculating costs/resource requirements. Future studies with sequential sampling of the same individuals over time may shed some light on the duration of ReA. In addition, reporting on the specifics of the questions used during follow-up could add clarity as to whether the study reported incident or prevalent cases.

The method of diagnosis for the sequelae was also a significant contributor to heterogeneity for cases of NTS and ReA. There was a large range of diagnostic criteria used within the physician/specialist confirmed studies. The lack of a clear definition for ReA [6] is problematic as it prevents meaningful comparison between studies. Moreover, the higher proportion of ReA cases for studies based on self-reported results indicate that personal perception may play a role in disease status. The wording and timing of questions investigating $\mathrm{ReA}$ could influence the differences seen in diagnostic method but with the limited details published in most studies it was not possible to investigate these effects further.

For IBS, several variables were significantly associated with heterogeneity in univariable analysis. However, it is difficult to interpret the results due to the low number of studies, and inter-relatedness between variables. Thus, further research is needed to accurately estimate the proportion developing IBS and the factors associated with this.

For ReA and IBS, heterogeneity remained high after subgroup analysis, suggesting that additional sources of heterogeneity are present. Some of the factors that were evaluated, such as age, were inconsistently reported in the primary studies, limiting a comprehensive evaluation of their impact on heterogeneity. Analyses of sources of heterogeneity, and the interpretation of those analyses was complicated by the inter-relatedness of many of the variables and the small sample size available for evaluating potential confounding or interaction between variables. For instance, surveillance studies also tended to be retrospective and generally also had the larger samples sizes. Additional sources of heterogeneity could be related to host, pathogen and environmental factors not evaluated in this study. For example the race, gender or immune status of sequelae cases, the dose of pathogen received, severity of acute illness and virulence of the Salmonella strain are all potential sources of heterogeneity that were not investigated. For many of these factors, the information is not easily ascertained from the published literature.
A number of the variables evaluated were not significantly associated with heterogeneity. However, given the small number of studies and low power to detect significant differences, non-significant results should be interpreted with caution.

There were several additional limitations to this study. A relatively large number of potentially relevant publications were not in English and resources were not available to translate these reports. It is not known the extent to which this would impact the results. Future studies should consider acquiring resources for translation or estimating country-specific estimates, if sufficient data are available. Categorization of group size and follow up time introduced the potential for bias as the categories were determined post-hoc. The assumption of independence of outcomes for the meta-analysis was not met as multiple outcomes were used from some studies. This in combination with the large amount of unidentified heterogeneity indicates the summary estimates reported in this study must be interpreted with caution.

Reporting of key features was not consistent in publications. This made interpretation of the results difficult. In particular, non-reporting of results by age meant that estimates could not be calculated within age group, limiting the usefulness of these results in burden of illness studies where measures such as DALYs calculate burden based on frequency of illness within age groups. Missing data also decreased power to detect significant sources of heterogeneity. Although the review included a range of study designs, guidelines for reporting research studies are available for many study designs, including observational studies (the STROBE statement) [50], surveys [51], and outbreaks (the ORION statement) [52]. Comprehensive and transparent reporting of research execution and results is essential to the use of those results for secondary purposes. Over time, use of guidelines could result in a more consistent body of data to inform burden of illness studies.

\section{CONCLUSION}

Estimates for the proportion of NTS cases developing ReA and IBS were calculated. However, extremely high heterogeneity in the estimates, likely due to differences in methodology between studies, limits the usefulness of these estimates. For more accurate estimates to be developed, consistent diagnostic approaches and case definitions need to be implemented and reported in future research. 


\section{ACKNOWLEDGEMENTS}

Funding for this research was received from a Canadian Institutes of Health Research (CIHR) Institute of Population and Public Health/Public Health Agency of Canada Applied Public Health Research Chair. In addition, financial and in-kind support was provided by the Public Health Agency of Canada. The authors thank Mai Pham, Ashley Whiteman, Dianna Wolfe, Tyler O'Neil and Theresa Procter for technical assistance with this study.

\section{DECLARATION OF INTEREST}

None.

\section{REFERENCES}

1. Majowicz SE, et al. The global burden of nontyphoidal Salmonella gastroenteritis. Clinical Infectious Diseases 2010; 50: 882-889.

2. Stevens MP, Humphrey TJ, Maskell DJ. Molecular insights into farm animal and zoonotic Salmonella infections. Biological Sciences 2009; 364: 2709-2723.

3. Fatica MK, Schneider KR. Salmonella and produce: survival in the plant environment and implications in food safety. Virulence 2011; 2: 573-579.

4. Foley SL, Lynne AM. Food animal-associated Salmonella challenges: pathogenicity and antimicrobial resistance. Journal of Animal Science 2008; 86: E173-87.

5. Townes JM. Reactive arthritis after enteric infections in the United States: the problem of definition. Clinical Infectious Diseases 2010; 50: 247-254.

6. Thabane M, Kottachchi DT, Marshall JK. Systematic review and meta-analysis: the incidence and prognosis of post-infectious irritable bowel syndrome. Alimentary Pharmacology \& Therapeutics 2007; 26: 535-544.

7. Thomas MK, et al. Estimated numbers of community cases of illness due to Salmonella, Campylobacter and verotoxigenic Escherichia coli: pathogen-specific community rates. Canadian Journal of Infectious Diseases \& Medical Microbiology 2006; 17: 229-234.

8. Scallan E, et al. Foodborne illness acquired in the United States - major pathogens. Emerging Infectious Diseases 2011; 17: 7.

9. Havelaar AH, et al. Disease burden of foodborne pathogens in the Netherlands, 2009. International Journal of Food Microbiology 2012; 156: 231-238.

10. Haagsma JA, et al. Systematic review of burden of foodborne illness studies: quality assessment of data and methodology. International Journal of Food Microbiology 2013; 166: 34-47.

11. Higgins JPT, Green S. Cochrane Handbook for Systematic Reviews of Interventions, version 5.1.0. The Cochrane Collaboration 2011; 5.1.0.

12. Keithlin J. A systematic review, meta-analysis and meta-regression of the proportion of Campylobacter, non-typhoidal Salmonella and E. coli O157 cases that develop chronic sequelae (thesis). Guelph, ON, Canada: University of Guelph, 2012, 176 pp.

13. Keithlin J, et al. Chronic sequelae of E. coli O157: systematic review and meta-analysis of the proportion of $\mathrm{E}$. coli 0157 cases that develop chronic sequelae. Foodborne Pathogens and Disease 2014; 11: 79-95.

14. Sanchez J, et al. Factors influencing the prevalence of Salmonella spp. in swine farms: a meta-analysis approach. Preventive Veterinary Medicine 2007; 81: $148-177$.

15. Higgins JP, Thompson SG. Quantifying heterogeneity in a meta-analysis. Statistics in Medicine 2002; 21: $1539-1558$

16. Borenstein M, et al. Introduction to Meta-analysis. United Kingdom: John Wiley and Sons, Ltd, 2009, pp. 421.

17. Karlinger K, et al. The epidemiology and the pathogenesis of inflammatory bowel disease. European Journal of Radiology 2000; 35: 154-167.

18. Arányi Z, et al. Miller Fisher syndrome: brief overview and update with a focus on electrophysiological findings. European Journal of Neurology 2012; 19: 1520.

19. Locht H, Molbak K, Krogfelt KA. High frequency of reactive joint symptoms after an outbreak of Salmonella enteritidis. Journal of Rheumatology 2002; 29: 767-771.

20. Thomson GT, et al. Post-Salmonella reactive arthritis: late clinical sequelae in a point source cohort. American Journal of Medicine 1995; 98: 13-21.

21. Ternhag A, et al. Short- and long-term effects of bacterial gastrointestinal infections. Emerging Infectious Diseases 2008; 14: 143-148.

22. Doorduyn Y, et al. Novel insight in the association between salmonellosis or campylobacteriosis and chronic illness, and the role of host genetics in susceptibility to these diseases. Epidemiology and Infection 2008; 136: $1225-1234$.

23. Ekman P, Kirveskari J, Granfors K. Modification of disease outcome in Salmonella-infected patients by HLA-B27. Arthritis and Rheumatism 2000; 43: 1527-1534.

24. Schiellerup P, Krogfelt KA, Locht H. A comparison of self-reported joint symptoms following infection with different enteric pathogens: effect of HLA-B27. Journal of Rheumatology 2008; 35: 480-487.

25. Townes JM, et al. Reactive arthritis following culture-confirmed infections with bacterial enteric pathogens in Minnesota and Oregon: a population-based study. Annals of the Rheumatic Diseases 2008; 67: 1689-1696.

26. Jess T, et al. Enteric Salmonella or Campylobacter infections and the risk of inflammatory bowel disease. Gut 2011; 60: 318-324.

27. Buxton JA, et al. Reactive arthritis and other sequelae following sporadic Salmonella typhimurium infection in British Columbia, Canada: a case control study. Journal of Rheumatology 2002; 29: 2154-2158.

28. Arnedo-Pena A, et al. Reactive arthritis and other musculoskeletal sequelae following an outbreak of 
Salmonella hadar in Castellon, Spain. Journal of Rheumatology 2010; 37: 1735-1742.

29. Cowden JM, et al. A national outbreak of Salmonella typhimurium DT 124 caused by contaminated salami sticks. Epidemiology and Infection 1989; 103: 219-225.

30. Dworkin MS, et al. Reactive arthritis and Reiter's syndrome following an outbreak of gastroenteritis caused by Salmonella enteritidis. Clinical Infectious Diseases 2001; 33: 1010-1014.

31. Eastmond CJ. Gram-negative bacteria and B27 disease. British Journal of Rheumatology 1983; 22: 67-74.

32. Hakansson U, et al. HL-A27 and reactive arthritis in an outbreak of salmonellosis. Tissue Antigens 1975; 6: 366-367.

33. Hannu T, et al. Reactive arthritis following an outbreak of Salmonella typhimurium phage type 193 infection. Annals of the Rheumatic Diseases 2002; 61: 264-266.

34. Locht H, Kihlstrom E, Lindstrom FD. Reactive arthritis after Salmonella among medical doctors - study of an outbreak. Journal of Rheumatology 1993; 20: 845-848.

35. Mattila L, et al. Reactive arthritis following an outbreak of Salmonella bovismorbificans infection. Journal of Infection 1998; 36: 289-295.

36. Mattila L, et al. Reactive arthritis following an outbreak of Salmonella infection in Finland. British Journal of Rheumatology 1994; 33: 1136-1141.

37. McColl GJ, et al. HLA-B27 expression and reactive arthritis susceptibility in two patient cohorts infected with Salmonella typhimurium. Australian and New Zealand Journal of Medicine 2000; 30: 28-32.

38. McKendrick MW, Read NW. Irritable bowel syndrome - post Salmonella infection. Journal of Infection 1994; 29: 1-3.

39. Mearin F, et al. Dyspepsia and irritable bowel syndrome after a Salmonella gastroenteritis outbreak: one-year follow-up cohort study. Gastroenterology 2005; 129: 98-104.

40. Rudwaleit M, et al. Low incidence of reactive arthritis in children following a Salmonella outbreak. Annals of the Rheumatic Diseases 2001; 60: 1055-1057.
41. Samuel MP, et al. Fast food arthritis - a clinicopathologic study of post-Salmonella reactive arthritis. Journal of Rheumatology 1995; 22: 1947-1952.

42. Thomson GT, et al. Immunoepidemiology of postSalmonella reactive arthritis in a cohort of women. Clinical Immunology and Immunopathology 1992; 64: 227-232.

43. Urfer E, et al. Outbreak of Salmonella braenderup gastroenteritis due to contaminated meat pies: Clinical and molecular epidemiology. Clinical Microbiology and Infection 2000; 6: 536-542.

44. Lee AT, Hall RG, Pile KD. Reactive joint symptoms following an outbreak of Salmonella typhimurium phage type 135a. Journal of Rheumatology 2005; 32: 524-527.

45. Rohekar S, et al. Symptomatic acute reactive arthritis after an outbreak of Salmonella. Journal of Rheumatology 2008; 35: 1599-1602.

46. Thomson GT, et al. Secretory immune response and clinical sequelae of Salmonella infection in a point source cohort. Journal of Rheumatology 1994; 21: 132-137.

47. Petersen AM, et al. Bacterial gastroenteritis among hospitalized patients in a Danish county, 1991-93. Scandinavian Journal of Gastroenterology 1996; 31: 906-911.

48. Saps M, et al. Post-infectious functional gastrointestinal disorders in children. Journal of Pediatrics 2008; 152: 812-816

49. Helms M, Simonsen J, Molbak K. Foodborne bacterial infection and hospitalization: a registry-based study. Clinical Infectious Diseases 2006; 42: 498-506.

50. von Elm E, et al. The Strengthening the Reporting of Observational Studies in Epidemiology (STROBE) statement: guidelines for reporting observational studies. Preventive Medicine 2007; 45: 247-251.

51. Kelley K, et al. Good practices in the conduct and reporting of survey research. International Journal for Quality in Healthcare 2003; 15: 261-266.

52. Stone SP, et al. The ORION statement: guidelines for transparent reporting of outbreak reports and intervention studies of nosocomial infection. Lancet Infectious Diseases 2007; 7: 282-288. 